\section{Revue de Sémantique et Pragmatique}

41-42 | 2017

Cadrage sur la variation, le changement lexical et le changement grammatical en français actuel

\title{
Un demi-siècle de conceptions du traitement de la variation dans la lexicographie d'expression française
}

Pierre Corbin et Nathalie Gasiglia

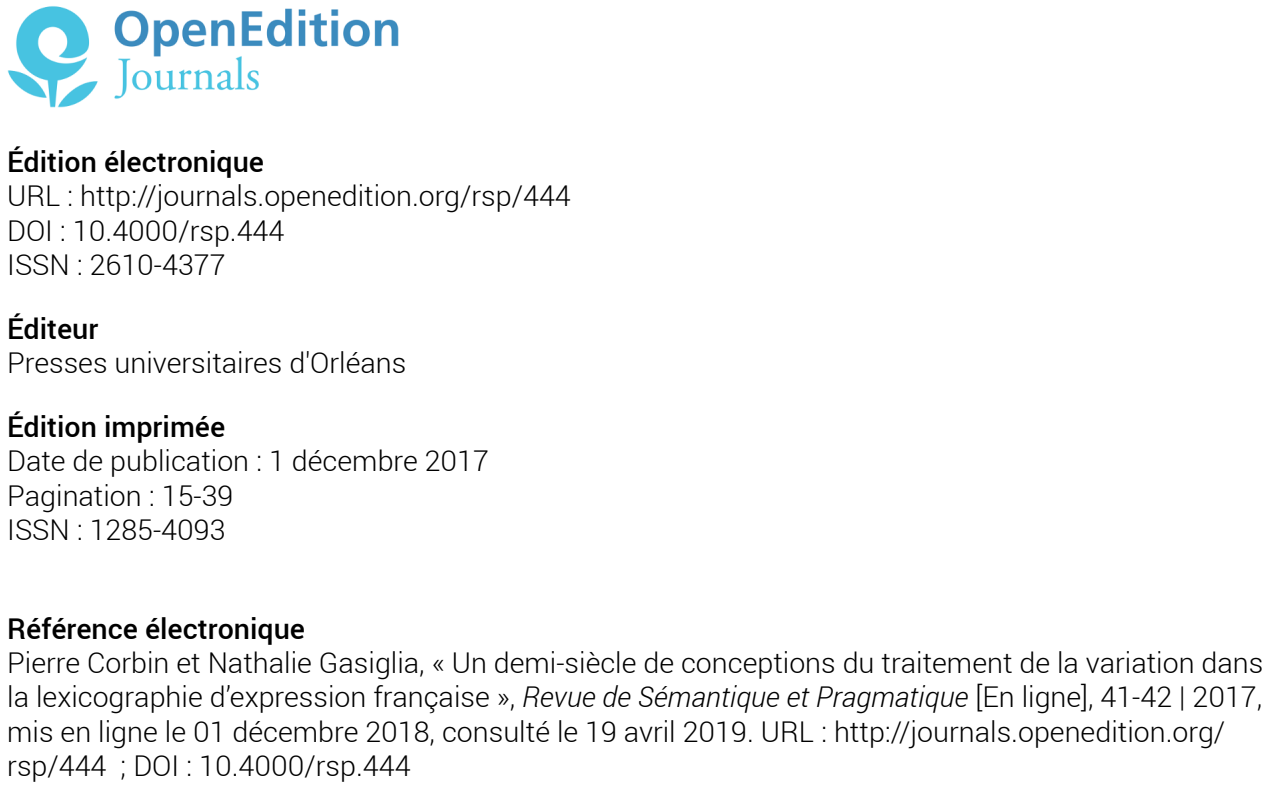

Revue de Sémantique et Pragmatique 
rs en linguis 
es (pour l'orthographe, le sens, la flexion, les constructions..., dans les nombreux répertoires utilitaires 
plois, en fonction des finalités définies pour chaque répertoire. Par exemple, le

ionalismes et mots de la francophonie », aux « domaines terminologiques » et aux « mots familiers et argotiques » appartenant au « vocabulaire courant », proposait systématiquement les «

isent des écarts par rapport

, pp. VI-VII.

variété de français identi 
é dans différents répertoires en fonction de paramètres définissant leur profil (projet, positionnement), se rencontre dans des préfaces qui évoquent des

bien constitué[s] », des « mots de valeur douteuse ou d'un emploi vicieux » visà-vis desquels le dictionnaire « met[...] [...] en garde [...] chaque fois que c'est nécessaire », des incidences lexicales des évolutions récentes des «diverses disciplines scientifiques » et de « toutes les branches de l'activité industrielle», des «mots familiers », des «mots étrangers admis dans la langue littéraire ou simplement technique », des «mots de sport » et des «mots dialectaux entrés dans l'usage ».

À sa suite, divers dictionnaires "encyclopédiques" et "de langue"

nt à des termes

liés aux innovations techniques, à des mots « dialectaux » et à des « néologismes

que" un répertoire qui conjoint

11) dénomme, un répertoire qui est supposé exclure les noms 
4), dont l'« Intro

gues particu

mes de « techniques artisanales [...] dépassées », au bénéfice de ceux des «

eillait « un certain

et même aux

mots grossiers en usage (assortis de « remarques normatives »), et accueillante

$$
\text { », p. [VI]. }
$$

iteur », t. I, p. V (évolution de l'«Avant-propos » de l'édition 
tière de sélection du lexique et de traitements qualifiant sa diversité. Pour

», que des explicitations, appuyées sur des

iques" ou "de langue", avec parfois d'importants

ges considérés comme «familier[s] », «populaire[s] » ou « argotique[s] », vis-à-vis desquels on souhaitait «multiplie[r] les indications sur les limitations d'emploi » (ce qui valait aussi pour des emplois considérés comme « litigieux » au regard du «bon usage », assortis à ce titre de «mises en garde ») 
iation, « niveaux de langue », qui réfère à un type d'information, était coordonné à « marques stylistiques », qui dénomme

enait le « niveau moyen de langue de l'homme cultivé » comme repère par rapport auquel appré mitamment, la « langue commune en usage aujourd'hui » comme étalon par rapport auquel qualifier comme « littéraires » ou " poétiques » des « emplois » évalués comme « trop aberrants »)

langue soutenue, littéraire » devait préciser « la situation de communication»

nettement que dans le

VII.

r Vincent Brugère-Trélat), $p$. IV. 
ation du dictionnaire » (signée par Alain Rey), respectivement pp. XX et

, pp. XXXIX-XL.

. 1, p. VI.

XIII.

uxième édition », t. 1, p. XL. 
du Petit Larousse », p. 7

e de la langue française », t. I, p. XII.

», p. [V].

ette.

aise », t. I, p. XII. 
loi $[\ldots] »$

ligatoire dans le cas marqué ("Régional", "Populaire", "Technique", "Vieux"). On appellera de telles

e et les marques stylistiques (fam., très fam., pop., argot ; ou

« Avant-propos », p. VII.

ctionnaire : mode d'emploi », pp. IV-V

Avant-propos », p. V. 
iquent le registre auquel appartient le mot. »

s rubriques connues : "familier", "populaire", "argot". »

) sont donnés avant la définition ou la construction s'il s'agit de sens généraux, immédiatement après la définition s'il s'agit d'expressions ou de la notation "langue soutenue". » 
tif et injurieux,

etc.) ; »

aines indications de « niveaux de langue ».

C'est dans les dictionnaires Robert que l'identification de variables aux

ge » typant des « valeurs d'emploi » étaient distribuées sur les cinq para

rs de « l'homogénéité du lexique » identifiés par Rey-Debove (1971 : 91) sous les dénominations d'«

ctivité »).

yé par « conditions d'emploi selon les situations » (

e dis

d'usage » du

« Marques

XVII. 
langue »)

ère seule étant associée à la notion de « niveau de

pour des usages spécialisés (§ 8.6)). S’y ajoutaient les marquages

la lexicographie française. On en trouve cepen 
dactique

$S \gg$

s l'inti

a »

-ci « sont au nombre de 264, dont 169 uniquement métropolitains, les 95 autres étant affectés d'un autre marqueur géographique » (cf. aussi Garnier 2008 : 58). Rapportés à l'ordre de grandeur de « 150000 définitions » affiché par l'éditeur (

s en vente au Canada, 60000 en Belgique,

ent similairement « rejetées » ( 
mation des principes de « représen

entent un inté

88, simultanément dans le

ette, qui était assorti d'un cahier de « mots français des francophones»

francophones

me édition », t. 1, p. XXIII.

os », p. V. 
la variété du français parlé en Afrique » sur les 111000 que

989, du centre Valibel (« Variétés linguistiques du français de Belgique »), préfigurant le

in » de l'INaLF, axée sur

ement africaine

de 1997, les valeurs annoncées étaient respectivement de 10000 définitions et 3000 noms propres provenant de «l'univers francophone » (quatrième de couverture ; cf. Corbin \& 
« l'un quelconque des "français régionaux" »

raphiques de la

e diaphasique "familier" à ce marquage diastratique destinée à traduire des évolutions des usages suscitées par les modifications du corps social et des modes de communication, mais induisant en retour un certain défi 
ssance fine des pratiques lexicales

crits, qui tireraient profit de la souplesse de paramétrage des affichages dans es, des initiatives 



ientifique 
es dictionnaires (6). Le commerce des mots », ann, O. Reichmann, H.E. Wiegand \& L. Zgusta (Hrsg.), guistique dans la formation et dans l'activité des lexicographes », in in, P. (2002), « Lexicographie et linguistique : une articulation difficile. L'exemple du domaine français », in F. Melka \& M. Celeste Augusto (eds),

004), « Théorie et pratique de la formation professionnelle de lexico

005), « Des occurrences discursives aux contextualisations dictionnai 
Gasiglia, N. (2010), « Représentations lexicographiques actuelles du lexique français : aperçus sur les choix de nomenclatures »,

lia, N. (2011), «Éléments pour un état de la description de la variété des usages lexicaux dans les dictionnaires français monolingues (1980-2008) », in

Corbin, P. \& Gasiglia, N. (2017), « "Ce qu'il faut mettre dans le dictionnaire” », in

rnier, Y. (2008), « Les francophonismes dans

is : options descriptives et choix rédactionnels », in J. Pruvost (dir.),

s fran

», in E. Bernal \& J. DeCesaris (eds), 
nolingues contempo

erung im allgemeinen einsprachigen Wörterbuch: eine Übersicht », in F.J. Hausmann, O. Reichmann, H.E. Wiegand \& L. Zgusta

description du français calédonien : état des lieux »,

nicas

nger (dir.),

phone ",

a structural dialectology possible?», 